\title{
Highly sensitive displacement sensor based on composite interference established within a balloon-shaped bent multimode fiber structure
}

\author{
Ke Tian \\ Harbin Engineering University \\ Gerald Farrell \\ Technological University Dublin, gerald.farrell@tudublin.ie \\ Xianfan Wang \\ Harbin Engineering University
}

See next page for additional authors

Follow this and additional works at: https://arrow.tudublin.ie/prcart

Part of the Electrical and Computer Engineering Commons

\section{Recommended Citation \\ Ke Tian, Gerald Farrell, Xianfan Wang, Elfed Lewis, and Pengfei Wang, "Highly sensitive displacement sensor based on composite interference established within a balloon-shaped bent multimode fiber structure," Appl. Opt. 57, 9662-9668 (2018), DOI: 10.1364/A0.57.009662}

This Article is brought to you for free and open access by the Photonics Research Centre at ARROW@TU Dublin. It has been accepted for inclusion in Articles by an authorized administrator of ARROW@TU Dublin. For more information, please contact arrow.admin@tudublin.ie, aisling.coyne@tudublin.ie,gerard.connolly@tudublin.ie.

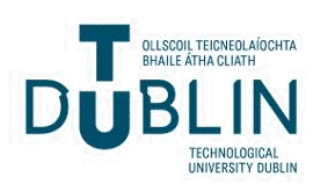


Authors

Ke Tian, Gerald Farrell, Xianfan Wang, Elfed Lewis, and Pengfei Wang

This article is available at ARROW@TU Dublin: https://arrow.tudublin.ie/prcart/27 


\title{
applied optics
}

\section{Highly sensitive displacement sensor based on composite interference established within a balloon-shaped bent multimode fiber structure}

\author{
Ke Tian, ${ }^{1}$ (i) Gerald Farrell, ${ }^{2}$ Xianfan Wang, ${ }^{1}$ Elfed Lewis, ${ }^{3}$ (i) and Pengfei Wang ${ }^{1,4, *}$ (i) \\ ${ }^{1}$ Key Laboratory of In-fiber Integrated Optics of Ministry of Education, College of Science, Harbin Engineering University, Harbin 150001, China \\ ${ }^{2}$ Photonics Research Centre, Dublin Institute of Technology, Kevin Street, Dublin 8, Ireland \\ ${ }^{3}$ Optical Fibre Sensors Research Centre, Department of Electronic and Computer Engineering, University of Limerick, Limerick, Ireland \\ ${ }^{4}$ Key Laboratory of Optoelectronic Devices and Systems of Ministry of Education and Guangdong Province, College of Optoelectronic \\ Engineering, Shenzhen University, Shenzhen 518060, China \\ ${ }^{*}$ Corresponding author: pengfei.wang@dit.ie
}

Received 4 July 2018; revised 30 September 2018; accepted 25 October 2018; posted 26 October 2018 (Doc. ID 337800); published 9 November 2018

\begin{abstract}
A novel optical fiber displacement sensor based on composite interference established within a balloon-shaped bent multimode (BSBM) fiber structure is described and experimentally demonstrated. The BSBM fiber structure is realized by bending a straight single-mode-multimode-single-mode (SMS) fiber structure into a balloon shape using a length of capillary tube to fix the shape of the structure. Owing to the bend in the multimode waveguide, the original undistorted multimode interference pattern is changed, and an extra Mach-Zehnder interferometer is effectively introduced within the multimode fiber (MMF) section at a suitable bending radius. This established composite interference greatly improves the displacement sensing performance of the SMS fiber structure. A maximum displacement sensitivity of $0.51 \mathrm{~dB} / \mu \mathrm{m}$ over the displacement range of $0-100 \mu \mathrm{m}$ at the operating wavelength of $1564.7 \mathrm{~nm}$ is achieved experimentally. Based on its easy fabrication process, low cost, and high measurement sensitivity, the sensor of this investigation could be a realistic candidate in the high-accuracy displacement measurement field. (๑) 2018 Optical Society of America
\end{abstract}

https://doi.org/10.1364/AO.57.009662

\section{INTRODUCTION}

Displacement measurement plays an important role in many areas with high-accuracy displacement sensors frequently required in many specialized application areas such as astronautics, microimaging, and structural health monitoring (SHM). Among the many kinds of displacement sensors, optical-fiber-based microdisplacement sensors have attracted significant research attention owing to their unique advantages of compact structure, the potential for remote operation, and inherent immunity to electromagnetic interference. Recently, a fiber-optic microdisplacement sensor based on surface plasmon resonance (SPR) has been reported in Ref. [1]; using interrogation in the wavelength domain, it possesses an excellent sensing performance featuring that an ultra-high displacement sensitivity of $10.32 \mathrm{~nm} / \mu \mathrm{m}$ was achieved experimentally. However, the preprocessing required for this type of sensing structure is rather complex in that it requires numerous technical steps including side-polishing, gold plating, and micromechanical operation. Such complex processing also results in timeconsuming and high-cost fabrication, leading to a high cost for a complete sensor system. Therefore, when considering practical fabrication requirements and cost, many other microdisplacement sensors based on structures such as a Mach-Zehnder interferometer (MZI) [2], a Sagnac interferometer [3], a fiber Bragg grating (FBG) [4], a long-period grating (LPG) [5], and a photonic crystal fiber (PCF) [6] are hardly widely used in current practical applications.

In recent years, single-mode-multimode-single-mode (SMS) fiber structures have been intensively investigated and widely employed in optical communications and sensing fields. A range of photonic devices have been successfully developed based on the multimode interference (MMI), which generally exists within multimode waveguides; examples include a wavelength division multiplexer [7], an edge filter [8], and a bandpass filter [9]. Given the excellent advantages of SMS fiber structures including compact size, low cost, and ease of fabrication, SMS fiber structures have also been developed as sensors for a wide range of measurands, e.g., strain [10], humidity [11], temperature $[12,13]$, curvature $[14,15]$, refractive index (RI) $[16,17]$, and magnetic field strength $[18,19]$. Displacement measurement also can be fulfilled by using SMS fiber structures. For example, a microdisplacement sensor based on the 
MMI effect with an achieved displacement sensitivity of $25 \mathrm{pm} / \mu \mathrm{m}$ has been reported in Ref. [20]. However, its sensing structure demands a relatively large effort with the inclusion of index matching liquid, which also significantly decreases the stability of the integrated sensor system. Another microdisplacement sensor based on a microbend SMS fiber structure has been reported in Ref. [21]; it can provide a useful displacement sensitivity at a relatively high displacement range (900$1000 \mu \mathrm{m})$. However, its sensing performance is not good enough at the relatively low displacement range $(0-200 \mu \mathrm{m})$ where a sensitivity of only $0.009 \mathrm{~dB} / \mu \mathrm{m}$ is achieved, using interrogation in the intensity domain. Therefore, there is a clear requirement to develop an SMS-based fiber structure displacement sensor that is robust and easy to fabricate, and can provide a higher measurement sensitivity than existing versions. In previous articles published by some of the authors of this paper, it has been demonstrated that the use of composite interference within the multimode fiber (MMF) can enhance the sensing performance of traditional SMS fiber structures. For example, an MZI can be introduced in the MMF section using torsion, and this twisted SMS fiber structure was proven to improve the curvature sensitivity of a traditional SMS fiber structure by almost 2 times [14]. In another scenario, a quasi-FabryPerot cavity was embedded within an SMS fiber structure and was proven to offer double the strain sensitivity compared to a traditional SMS fiber structure [10].

In this paper, a novel optical fiber displacement sensor based on composite interference established within a balloon-shaped bent multimode (BSBM) fiber structure is demonstrated. The schematic configuration of the sensor is shown in Fig. 1. The BSBM fiber structure is simply fabricated by bending the multimode section of an SMS fiber structure into "balloon shape" using a length of a capillary tube. With the introduction of a bend in the multimode waveguide, the nature of the original MMI is changed and, furthermore, an extra MZI can be introduced within MMF section at a suitable bending radius, creating a composite interference. This established composite interference is analyzed theoretically, and the actual displacement sensing performance and also the temperature dependence of the sensor are investigated experimentally. Benefiting from its excellent advantages of low-cost, simple fabrication, and experimentally demonstrated high sensitivity associated with good repeatability, this displacement sensor could be a competitive candidate for accurate displacement measurement in a range of areas.

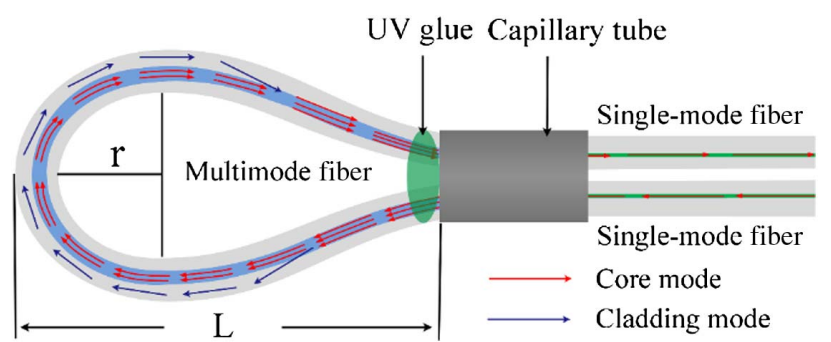

Fig. 1. Schematic diagram of the balloon-shaped bent MMF structure.

\section{PRINCIPLE AND ANALYSIS}

Figure 1 shows a schematic illustration of the light propagation schematic in a balloon-shaped bent SMS fiber structure. The bending radius and the width of the BSBM fiber structure are defined as $r$ and $L$, respectively. The interconnecting splices from/to the SMF fibers lie within the capillary. This means that as shown in the figure, the MMF section has both a strongly bent balloon-shaped subsection as well as two relatively straight subsections.

As shown in Fig. 1, the light from the source initially propagates along the input SMF, and when the light launches into the MMF section, a series of high-order modes are excited. For a step-index MMF, the number of excited modes $M$ can be approximately calculated by [22]

$$
M \approx \frac{2 a}{\lambda} \sqrt{n_{\mathrm{co}}^{2}-n_{\mathrm{cl}}^{2}}
$$

where $a$ is the radius of the MMF core, $n_{\mathrm{co}}$ and $n_{\mathrm{cl}}$ are the RI for the MMF core and cladding, respectively, and $\lambda$ is the free space wavelength. When the light arrives at the balloon-shaped subsection, owing to the bend in the multimode waveguide, a portion of the light from the MMF core is coupled into the cladding at which point it excites cladding modes. These excited cladding modes propagate within the MMF cladding, and following propagation through the bend region, a portion of the cladding mode energy is recoupled back into the MMF core. Interference between the cladding modes and the modes propagating in the core occurs, and thus an MZI is formed due to the existence of differences in effective RIs of the core and the cladding and the optical path differences experienced by the light signals propagating in the core modes and the cladding modes. In the case of a typical two-mode MZI, the output intensity can be expressed as follows [23]:

$$
I_{\text {out }}=I_{\mathrm{co}}^{m}+I_{\mathrm{cl}}^{n}+2 \sqrt{I_{\mathrm{co}}^{m} I_{\mathrm{cl}}^{n}} \cos \left(\varphi+\varphi_{0}\right),
$$

where $I_{\mathrm{co}}^{m}$ and $I_{\mathrm{cl}}^{n}$ are the intensity of the $m$ th core mode and $n$th cladding mode, and $\varphi_{0}$ is the initial phase. The phase difference $\varphi^{m n}$ between the $m$ th core mode and the $n$th cladding mode can be expressed as [24]

$$
\varphi^{m n}=\frac{2 \pi L_{\mathrm{eff}}}{\lambda} \Delta n_{\mathrm{eff}}^{m n},
$$

where $L_{\text {eff }}$ is the effective bent length, and $\Delta n_{\text {eff }}^{m n}=n_{\mathrm{co}}^{m}-n_{\mathrm{cl}}^{n}$ is the effective RI difference between the core modes and the cladding modes. A detailed theoretical analysis of the MMF-based MZI pattern is presented in Ref. [25].

Additionally, the bend also has an effect on the physical RI of the fiber because of the elasto-optical effect, significantly influencing the MMI within the MMF. For a bent MMF, the RI distribution is no longer symmetrical. The RI distribution within a bent MMF can be expressed in terms of that in a straight MMF and is expressed as [26]

$$
n^{\prime}=n_{0}\left(1+\frac{x}{r_{\text {eff }}}\right)
$$

where $n_{\mathrm{o}}$ is the RI for the straight fiber section, $x$ is the normal axis to the bent fiber, and $r_{\text {eff }}$ is the equivalent bend radius, which can be calculated by [21] 
(a)

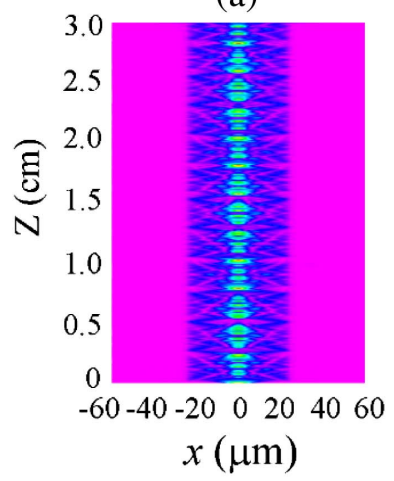

1.0

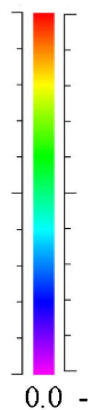

(b)

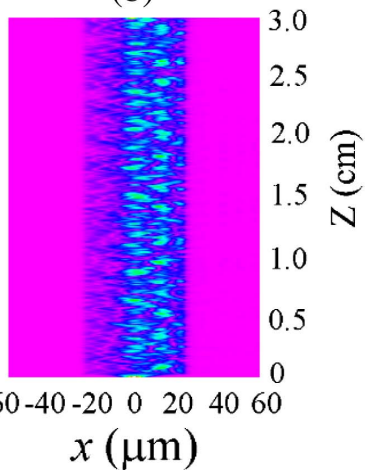

Fig. 2. Simulated optical field intensity spatial distribution at a wavelength of $1550 \mathrm{~nm}$ (a) within a straight MMF section and (b) within a bent MMF section.

$$
r_{\text {eff }}=\frac{r}{1-\frac{n_{0}^{2}}{2}\left[P_{12}-v\left(P_{11}+P_{12}\right)\right]},
$$

where $r$ is the actual bend radius of fiber, $v$ is the Poisson ratio, and $P_{11}$ and $P_{12}$ are the components' photoelastic tension.

Numerical simulations have been performed using a commercial software package Rsoft based on the beam propagation method (BPM) for the MMI in the case of the straight and the bent MMF. To simplify the simulation process, only the most curved section (the top of the balloon-shaped section) was considered because this section has a constant bend radius. The numerical mesh size in the profile directions $X, Y$, and propagation direction $Z$ were $0.1 \mu \mathrm{m}, 0.1 \mu \mathrm{m}$, and $1 \mu \mathrm{m}$, respectively. The simulation window size was $130 \mu \mathrm{m} \times$ $30,000 \mu \mathrm{m}$, and the boundary condition corresponded to a perfectly matched layer condition. The incident light intensity profile was assumed to be Gaussian, and the specific simulation parameters that were used for the diameter and RI for the MMF core and cladding were 50/125 $\mu \mathrm{m}$ and 1.4446/ 1.4271 , respectively. The length and the bending radius of the MMF used in the simulation are taken as 3 and $9.60 \mathrm{~mm}$, derived from simple initial experiments. When the free space wavelength was set to $1550 \mathrm{~nm}$, the calculated optical field distribution results for the straight and the bent MMF are presented in Fig. 2(a) and 2(b), respectively. It is worth noting that the actual bent MMF simulation model was established in a straight MMF but possesses an asymmetric RI distribution characteristic as defined in Eq. (4). As shown in Fig. 2(a), when the multimode waveguide is straight, the field distribution is symmetric in the radial direction of the MMF section. However, when the multimode waveguide is bent (the bending direction in the simulation model is toward the left in Fig. 2), the field distribution is no longer symmetrical; this is clearly evident in Fig. 2(b) since the RI distribution within the bent fiber is asymmetric as indicated by Eq. (4). Thus, the MMI within the bent MMF is significantly changed, which in turn influences the transmission characteristics of the SMS fiber structure.

Prior to the more formal experimental investigation described in the next section and based on the above theoretical analysis and numerical simulations, the transmission spectrum $(1500-1600 \mathrm{~nm})$ of the BSBM fiber structure with different bending radii was observed experimentally. Here, the length of the MMF section is carefully chosen to be $9.9 \mathrm{~cm}$. A value in this region was selected as for a typical SMS, reimaging lengths that at a periodic multiple of circa $4.45 \mathrm{~cm}$ are commonly found in the literature. From the measured transmission spectrum in Fig. 3, it is found that there exist some MMI induced dips for the straight SMS fiber structure. Also, as analyzed in Section 2, when the MMF is bent, composite interference can be potentially established. However, an appropriate bend radius needs to be determined to maximize this resultant interference. The bend radius was therefore decreased from 10.5 to $8.5 \mathrm{~mm}$ with a small step decrement of $0.5 \mathrm{~mm}$. When the bending radii are 10.5 and $10 \mathrm{~mm}$, no clear composite interference dips appear because too little light is coupled into the MMF cladding, and as a result the induced MZI is too weak to be observed with the measurement instrumentation available. As the bend radius was further decreased to $9.5 \mathrm{~mm}$, an enhanced interference pattern was obtained since the light energy distribution in the core and cladding is balanced, in which an interference dip at $1524.1 \mathrm{~nm}$ was observed with a relatively large extinction ratio (more than $18 \mathrm{~dB}$ ). As the bending radius continues to decrease to $9 \mathrm{~mm}$ or less than $8.5 \mathrm{~mm}$, the clarity of the interference fringe

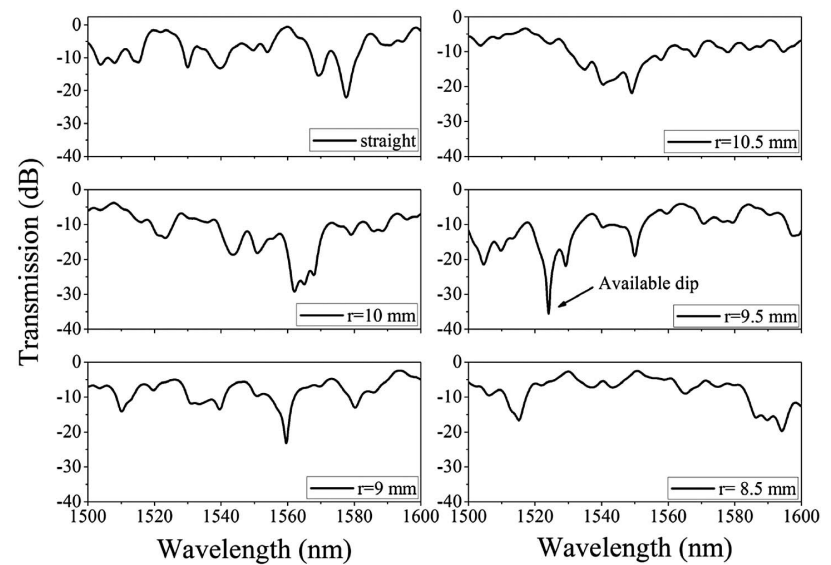

Fig. 3. Transmission spectrum of the BSBM fiber structure with different bending radii. 
becomes low compared to the $9.5 \mathrm{~mm}$ bend radius case. This is most likely because with decreasing bend radius, an excessive proportion of the light is coupled from the core into the cladding, resulting in a weak MMI. Ultimately, these initial exploratory experiments determined that the appropriate bending radius to form an effective bent MMF section with a usable interference dip should be close to $9.5 \mathrm{~mm}$.

\section{EXPERIMENTS AND DISCUSSION}

To fabricate a robust and mechanically stable BSBM fiber structure, a conventional SMS fiber structure was first constructed by splicing a $9.9 \mathrm{~cm}$ length of MMF (AFS 50/125, Thorlabs) with two standard single-mode fibers (SMF-28). Next, both ends of the fabricated SMS fiber structure were inserted into a capillary tube for which the length and inner diameter are $1.5 \mathrm{~cm}$ and $600 \mu \mathrm{m}$, respectively. By moving this capillary tube along the fiber, the bending radius of the balloon-shaped section can be easily and finely adjusted. After the optimal bending radius was determined, UV glue was used to immobilize the BSBM fiber structure. The entire fabrication process for the BSBM fiber structure is simple. The fabricated sample has a measured bending radius $r$ value of $9.60 \mathrm{~mm}$ (the bending radius was adjusted to $9.60 \mathrm{~mm}$ to achieve an optimum extinction ratio of the interference dips), and the corresponding width of the balloon-shaped subsection $L$ value is $35.93 \mathrm{~mm}$. The transmission spectrum of the fabricated sample was measured and is depicted in Fig. 4. It is clear that as expected there exists a desired interference dip over the wavelength rang of 1500-1600 $\mathrm{nm}$ with a central wavelength located at $1564.7 \mathrm{~nm}$. This dip was chosen to perform displacement measurement since it has a relatively high extinction ratio $(21.40 \mathrm{~dB})$.

The experimental setup for displacement measurement is shown in Fig. 5. The sample was placed and fixed on a lifting platform, which was height adjustable. A one-dimensional manually tuned translation stage with a $10 \mu \mathrm{m}$ displacement adjustment was used to accurately change the axial displacement, which was applied to the balloon-shaped section. A supercontinuum light source (SCS, YSL SC-series, China) was connected to the input SMF of the sensor, and the output transmission spectrum was recorded in real-time using a

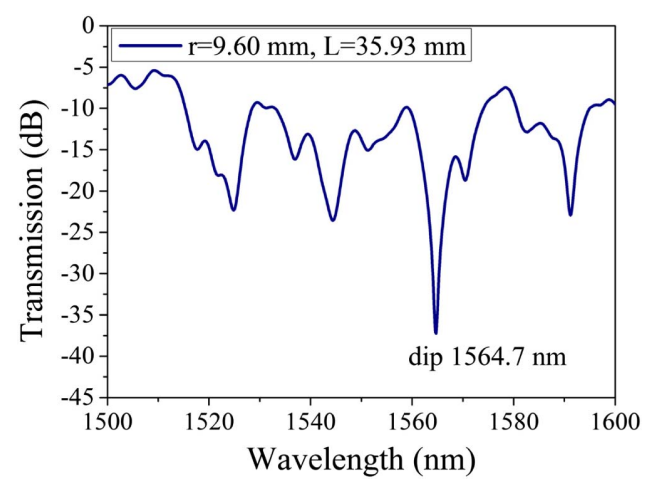

Fig. 4. Transmission spectrum of the BSBM fiber structure sample with the bending radius of $9.60 \mathrm{~mm}$ and the width of the balloonshaped section of $35.93 \mathrm{~mm}$.

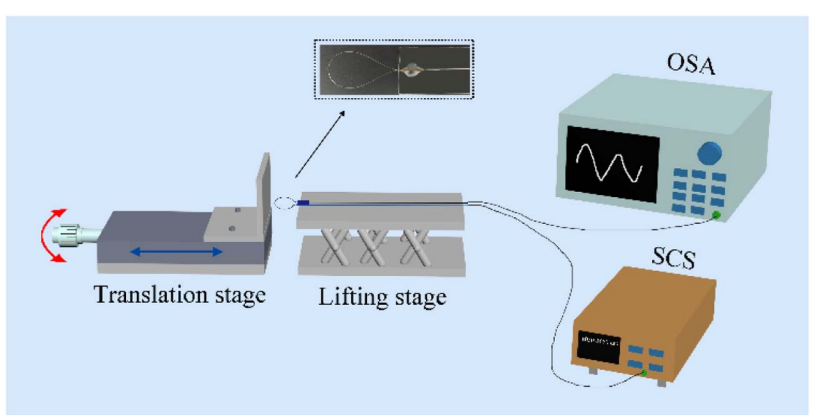

Fig. 5. Schematic diagram of the experimental setup for displacement measurement using the proposed sensor.

high-resolution $(20 \mathrm{pm})$ optical spectrum analyzer (OSA, YOKOGAWA AQ6370D, Japan).

The transmission spectrum evolution of the proposed fiber structure when subject to a displacement from $0-100 \mu \mathrm{m}$ with a step of $10 \mu \mathrm{m}$ is depicted in Fig. 6(a). To test the repeatability of the sensor, a reversed measurement cycle was carried out in which the displacement was decreased from $100 \mu \mathrm{m}$ back to $0 \mu \mathrm{m}$, and the result is depicted in Fig. 6(b). As shown in Fig. 6(a), when the displacement increased, the dip of $1564.7 \mathrm{~nm}$ experiences a blue shift and was accompanied by a decrease in the extinction ratio of the dip. When the displacement changed direction, the spectral evolution is reversed,
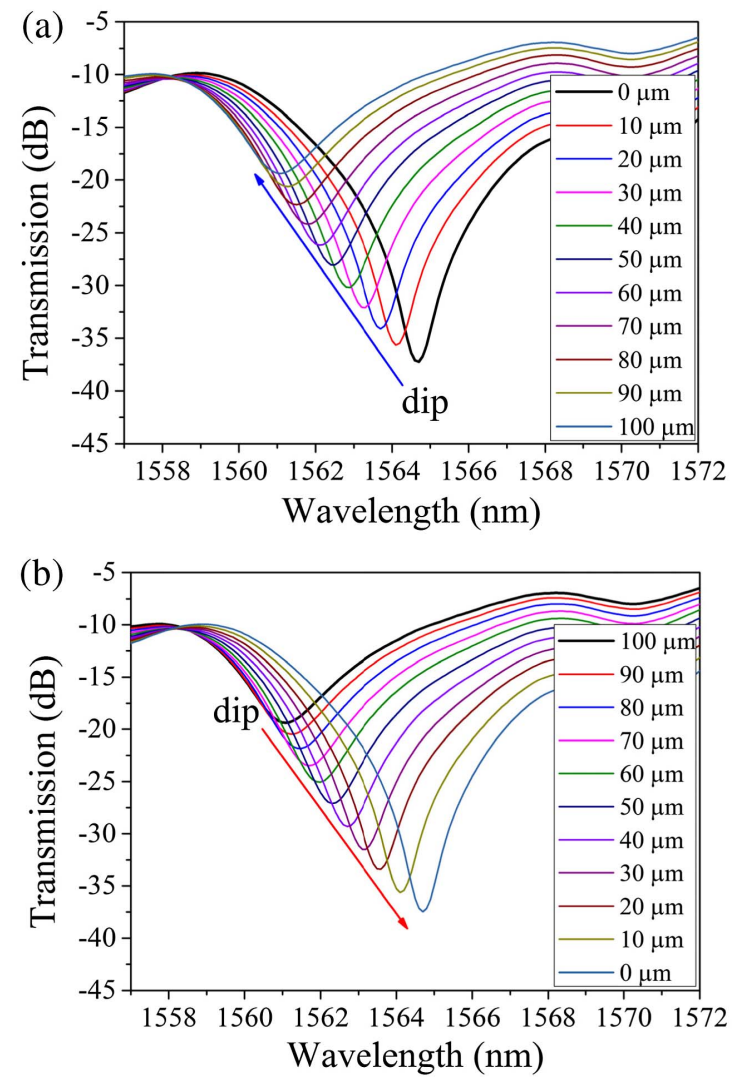

Fig. 6. Dip evolution when applied displacement changes: (a) displacement increases $(0-100 \mu \mathrm{m})$; (b) displacement decreases (100$0 \mu \mathrm{m})$. 
which is clearly shown in Fig. 6(b). The fact that both the dip wavelength and the intensity changes is due to the displacement-induced shape change of the balloon-shaped section that ultimately results in the variation of the effective bent length $L_{\text {eff }}$ and the light coupling between the MMF core and cladding.

The resulting wavelength shift and intensity change of the dip centered on $1564.7 \mathrm{~nm}$ with increasing and decreasing displacement are plotted and linearly fitted in Fig. 7(a) and 7(b), respectively. From Fig. 7(a), the wavelength-displacement sensitivities of the $1564.7 \mathrm{~nm}$ dip for the displacement increasing and decreasing processes were calculated to be -36 and $-36.4 \mathrm{pm} / \mu \mathrm{m}$, respectively with a linear regression coefficient value $\left(R^{2}\right)$ of 0.9824 and 0.9732 . From Fig. $7(\mathrm{~b})$, the intensitydisplacement sensitivities of the $1564.7 \mathrm{~nm}$ dip for the displacement increasing and decreasing processes were calculated to be 0.186 and $0.189 \mathrm{~dB} / \mu \mathrm{m}$ with an $R^{2}$ of 0.9980 and 0.9932 . Based on the tiny difference between the results for the cases of increasing and decreasing displacement, it is also evident that there is no hysteresis present and that this sensor has a good repeatability for displacement measurement. Furthermore, given the $20 \mathrm{pm}$ resolution OSA used in the experiment, the displacement measurement resolution based on wavelength interrogation system is estimated to be $0.56 \mu \mathrm{m}$.

From the observed dip evolution features, a simple intensity/power interrogation system can be adopted to measure displacement by means of purely intensity change. This means
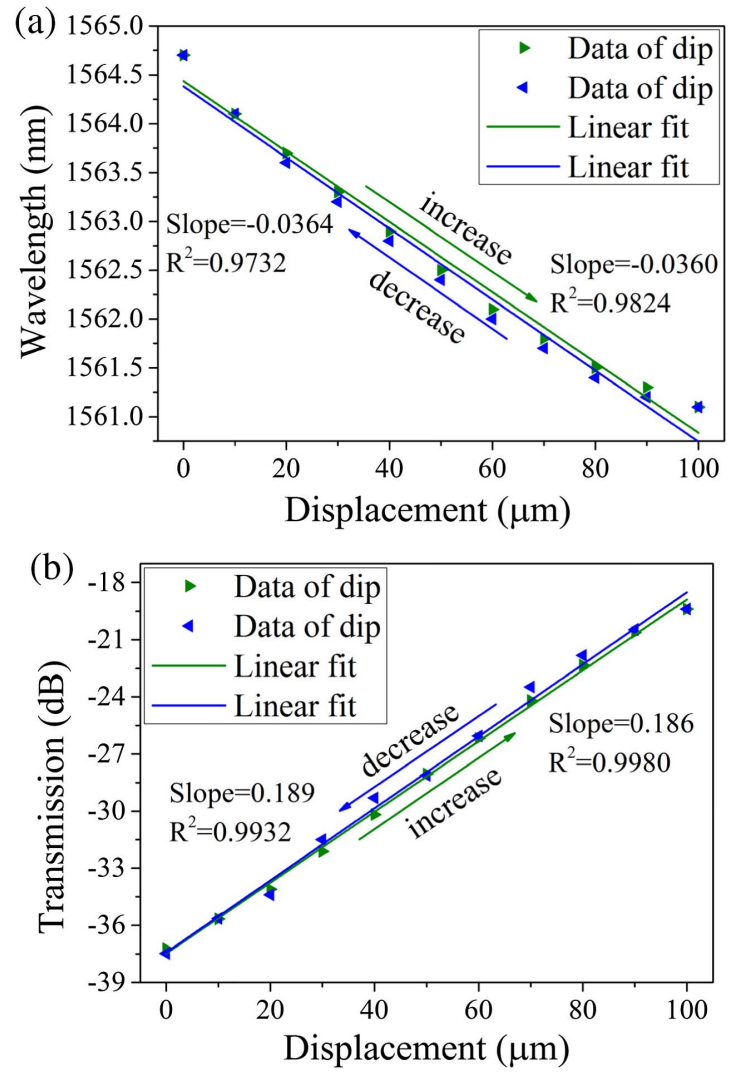

Fig. 7. Spectrum variation as a function of displacement including displacement increases and decreases processes (a) dip at wavelength change and (b) dip at intensity change.
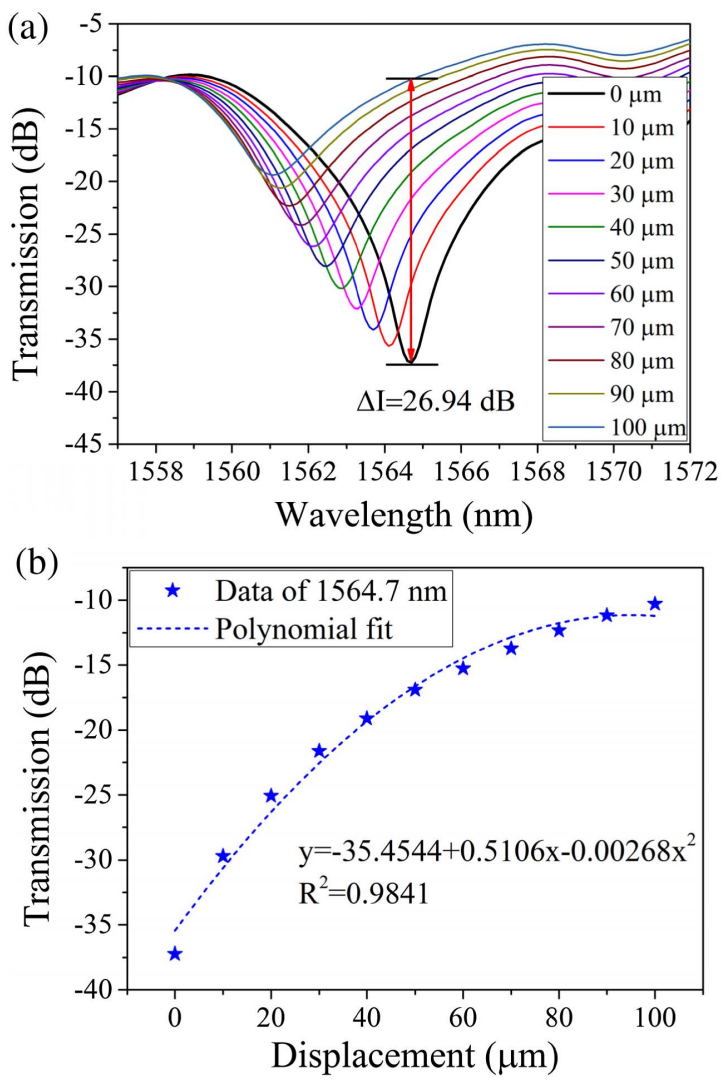

Fig. 8. (a) Intensity change of a single wavelength of $1564.7 \mathrm{~nm}$ when applied displacement changed; (b) polynomial fitting result.

the expensive OSA and SCS can be replaced with a standard power meter or photoamplifier and a fixed wavelength LED or laser light source with an appropriate choice of wavelength, which greatly reduces the cost of the entire sensor system. For an intensity/power interrogation system, it is important to determine the monitoring wavelength, because the measurement sensitivity will vary for different wavelengths. From Fig. 8(a), it is easily observed that the maximal intensity change is achieved at $1564.7 \mathrm{~nm}$ (the central wavelength of the chosen dip) for which the intensity change reaches $26.94 \mathrm{~dB}$ as the displacement changes from 0 to $100 \mu \mathrm{m}$. The operating wavelength was therefore chosen at this wavelength to obtain a maximum intensity-displacement sensitivity. The corresponding intensity variation against displacement change was plotted and fitted with a polynomial fitting function as depicted in Fig. 8(b). The fitting results predict that the intensity-displacement sensitivity is as high as $0.51 \mathrm{~dB} / \mu \mathrm{m}$ with an $R^{2}$ of 0.9841 . Compared to the other configurations based on displacement sensors that also utilize the intensity/ power interrogation system cited in this work, the achieved sensitivity by the BSBM fiber structure of this investigation is 212 times and 56 times higher than the sensitivity values reported in Refs. [6] and [21], respectively. Furthermore, assuming the $0.01 \mathrm{~dB}$ resolution of a common power meter, it is possible to predict that using an intensity-based interrogation scheme, a displacement resolution of $0.02 \mu \mathrm{m}$ can be achieved. 


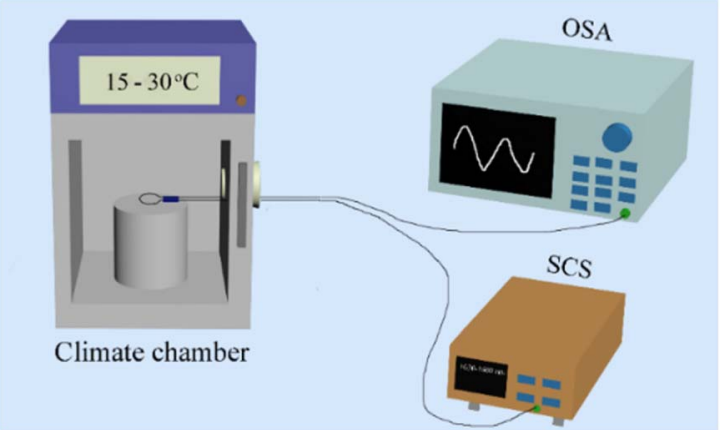

Fig. 9. Schematic diagram of the experimental setup for temperature measurement using the proposed sensor.

In addition to the displacement measurement, the temperature dependence of the proposed BSBM fiber structure was also investigated by placing the sensor sample inside a climate chamber (ESPEC SH-222, Japan), in which the temperature can be accurately controlled, and the real-time temperature value is shown on its external display panel. The previously mentioned SCS and OSA were used as the light source and detector, respectively. The corresponding experimental setup is schematically depicted in Fig. 9.

Owing to the limit imposed by the working temperature range of the UV glue, the sensor sample was subjected to a temperature change in the range of $15^{\circ} \mathrm{C}-30^{\circ} \mathrm{C}$ with a step
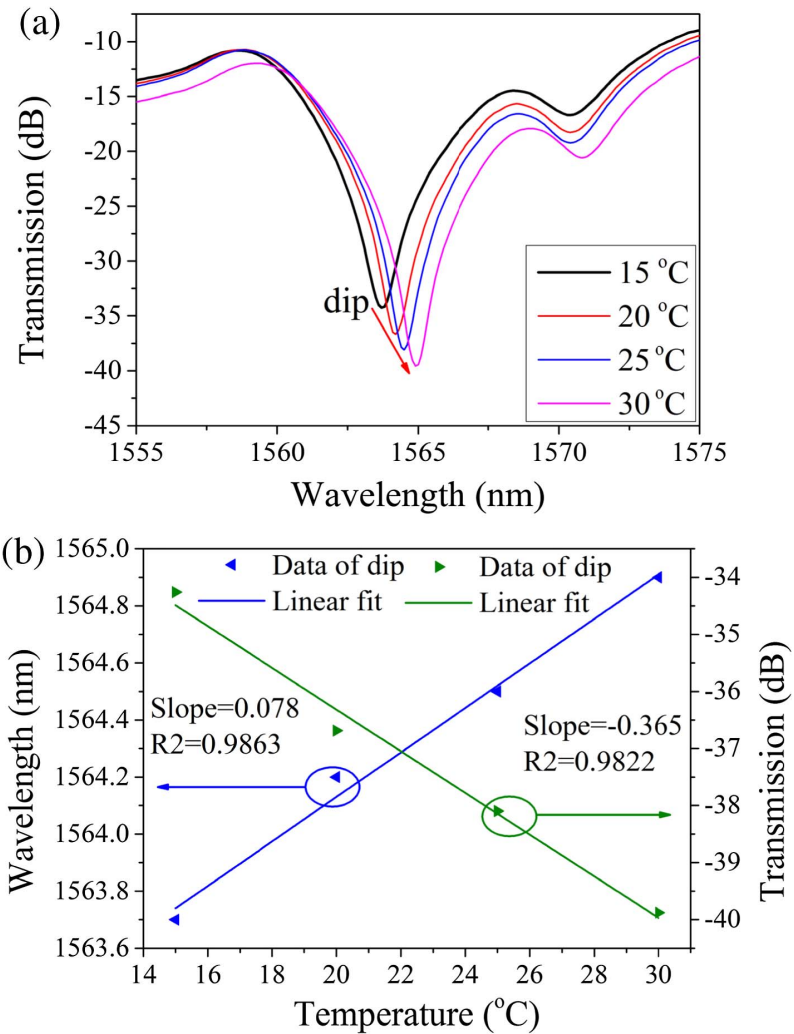

Fig. 10. (a) Dip evolution when the surrounding temperature changed; (b) dip shift as a function of temperature change; the blue line and the green line represent the wavelength-temperature and intensity-temperature fitting lines, respectively. of $5^{\circ} \mathrm{C}$. Figure 10 (a) shows the dip evolution result; it can be seen that the selected dip red shifts to longer wavelengths accompanied by an intensity (transmission) decrease when the temperature was increased. The measured data is plotted and fitted in Fig. 10(b). From the linear fitting results, the fitting curve of the wavelength temperature and intensity temperature both exhibit a high $R^{2}$ of 0.9863 and 0.9822 , and the corresponding temperature sensitivities were determined as $78 \mathrm{pm} /{ }^{\circ} \mathrm{C}$ and $-0.365 \mathrm{~dB} /{ }^{\circ} \mathrm{C}$, respectively. Under this circumstance, the temperature would need to be kept constant to better than $0.5^{\circ} \mathrm{C}$ during the displacement measurement.

\section{CONCLUSION}

In this paper, a high sensitivity optical fiber displacement sensor based on a BSBM fiber structure has been described. The sensor was simply realized by bending a straight SMS fiber structure into a balloon shape using a length of a capillary tube to fix the structure in place. Owing to the resulting large bending angle of the multimode waveguide, an effective composite interference pattern based on the changed MMI and the newly introduced MZI was established within the MMF section at a certain bending radius. The composite interference greatly improves the sensing performance of an SMS fiber structure compared to the unbent versions, with a maximum displacement sensitivity of $0.51 \mathrm{~dB} / \mu \mathrm{m}$ over the displacement range $0-100 \mu \mathrm{m}$ achieved at the operation wavelength of $1564.7 \mathrm{~nm}$. In conclusion, this low-cost and easily fabricated displacement sensor provides an experimentally proven high sensitivity and good repeatability, and it is envisioned that it could be a competitive candidate in the high-accuracy displacement measurement field.

Funding. National Key R\&D Program of China (2016YFE0126500); National Natural Science Foundation of China (NSFC) (61575050); Key Program for Natural Science Foundation of Heilongjiang Province (ZD2016012); Open Fund of the State Key Laboratory on Integrated Optoelectronics (SKLIOE) (IOSKL2016KF03); 111 project (B13015); Ph.D. Student Research and Innovation Fund of the Fundamental Research Funds for the Central Universities (HEUGIP201820); Government of Ireland International Scholarship programme.

\section{REFERENCES}

1. Z. Zhu, L. Liu, Z. Liu, Y. Zhang, and Y. Zhang, "High-precision micro-displacement optical-fiber sensor based on surface plasmon resonance," Opt. Lett. 42, 1982-1985 (2017).

2. J. Chen, J. Zhou, and Z. Jia, "High-sensitivity displacement sensor based on a bent fiber Mach-Zehnder interferometer," IEEE Photon. Technol. Lett. 25, 2354-2357 (2013).

3. M. Bravo, A. Pinto, M. Lopez-Amo, J. Kobelke, and K. Schuster, "High precision micro-displacement fiber sensor through a suspended-core Sagnac interferometer," Opt. Lett. 37, 202-204 (2012).

4. W. Zhang, X. Dong, Q. Zhao, and G. Kai, "FBG-type sensor for simultaneous measurement of force (or displacement) and temperature based on bilateral cantilever beam," IEEE Photon. Technol. Lett. 13, 1340-1342 (2001).

5. L. Qi, C.-L. Zhao, Y. Wang, J. Kang, Z. Zhang, and S. Jin, "Compact micro-displacement sensor with high sensitivity based on 
a long-period fiber grating with an air-cavity," Opt. Express 21, 31933200 (2013).

6. B. Dong and E. J. Hao, "Temperature-insensitive and intensitymodulated embedded photonic-crystal-fiber modal-interferometerbased microdisplacement sensor," J. Opt. Soc. Am. B 28, 2332-2336 (2011).

7. L. Han, S. Liang, J. Xu, L. Qiao, H. Zhu, and W. Wang, "Simultaneous wavelength-and mode-division (De) multiplexing for high-capacity onchip data transmission link," IEEE Photon. J. 8, 7903510 (2016).

8. A. M. Hatta, Y. Semenova, G. Rajan, and G. Farrell, "Polarization dependence of an edge filter based on singlemode-multimode-singlemode fibre," Opt. Laser Technol. 42, 1044-1048 (2010).

9. W. S. Mohammed, P. W. Smith, and X. Gu, "All-fiber multimode interference bandpass filter," Opt. Lett. 31, 2547-2549 (2006).

10. K. Tian, G. Farrell, X. Wang, W. Yang, Y. Xin, H. Liang, E. Lewis, and $P$. Wang, "Strain sensor based on gourd-shaped single-mode-multimode-single-mode hybrid optical fibre structure," Opt. Express 25, 18885-18896 (2017).

11. X. Wang, G. Farrell, E. Lewis, K. Tian, L. Yuan, and P. Wang, "A humidity sensor based on a singlemode-side polished multimodesinglemode optical fibre structure coated with gelatin," J. Lightwave Technol. 35, 4087-4094 (2017).

12. P. Wang, M. Ding, L. Bo, C. Guan, Y. Semenova, Q. Wu, G. Farrell, and G. Brambilla, "Fiber-tip high-temperature sensor based on multimode interference," Opt. Lett. 38, 4617-4620 (2013).

13. M. A. Fuentes-Fuentes, D. A. May-Arrioja, J. R. Guzman-Sepulveda, M. Torres-Cisneros, and J. J. Sánchez-Mondragón, "Highly sensitive liquid core temperature sensor based on multimode interference effects," Sensors 15, 26929-26939 (2015).

14. K. Tian, Y. Xin, W. Yang, T. Geng, J. Ren, Y.-X. Fan, G. Farrell, E. Lewis, and $P$. Wang, "A curvature sensor based on twisted single-mode-multimode-single-mode hybrid optical fiber structure," J. Lightwave Technol. 35, 1725-1731 (2017).

15. O. Frazão, J. Viegas, P. Caldas, J. Santos, F. Araújo, L. Ferreira, and F. Farahi, "All-fiber Mach-Zehnder curvature sensor based on multimode interference combined with a long-period grating," Opt. Lett. 32, 3074-3076 (2007)
16. Q. Wang and G. Farrell, "All-fiber multimode-interference-based refractometer sensor: proposal and design," Opt. Lett. 31, 317-319 (2006).

17. P. Wang, G. Brambilla, M. Ding, Y. Semenova, Q. Wu, and G. Farrell, "High-sensitivity, evanescent field refractometric sensor based on a tapered, multimode fiber interference," Opt. Lett. 36, 2233-2235 (2011).

18. Y. Chen, Q. Han, T. Liu, X. Lan, and H. Xiao, "Optical fiber magnetic field sensor based on single-mode-multimode-single-mode structure and magnetic fluid," Opt. Lett. 38, 3999-4001 (2013).

19. W. Lin, Y. Miao, H. Zhang, B. Liu, Y. Liu, and B. Song, "Fiber-optic inline magnetic field sensor based on the magnetic fluid and multimode interference effects," Appl. Phys. Lett. 103, 151101 (2013).

20. J. Antonio-Lopez, P. LiKamWa, J. Sanchez-Mondragon, and D. MayArrioja, "All-fiber multimode interference micro-displacement sensor," Meas. Sci. Technol. 24, 055104 (2013).

21. Q. Wu, Y. Semenova, P. Wang, A. M. Hatta, and G. Farrell, "Experimental demonstration of a simple displacement sensor based on a bent single-mode-multimode-single-mode fiber structure," Meas. Sci. Technol. 22, 025203 (2011).

22. Q. Wang, G. Farrell, and W. Yan, "Investigation on single-modemultimode-single-mode fiber structure," J. Lightwave Technol. 26, 512-519 (2008).

23. H. Y. Choi, M. J. Kim, and B. H. Lee, "All-fiber Mach-Zehnder type interferometers formed in photonic crystal fiber," Opt. Express 15, 5711-5720 (2007).

24. Y. Zhao, X. Liu, R.-Q. Lv, and Q. Wang, "Simultaneous measurement of $\mathrm{Rl}$ and temperature based on the combination of Sagnac loop mirror and balloon-like interferometer," Sens. Actuators B Chem. 243, 800-805 (2017).

25. C.-H. Chen, W.-T. Wu, and J.-N. Wang, "All-fiber microfluidic multimode Mach-Zehnder interferometers as high sensitivity refractive index sensors," Microsyst. Technol. 23, 429-440 (2017).

26. R. T. Schermer and J. H. Cole, "Improved bend loss formula verified for optical fiber by simulation and experiment," IEEE J. Quantum Electron. 43, 899-909 (2007). 